\title{
Cracks detection using active modal damping and piezoelectric components
}

\author{
B. Chomette ${ }^{\mathrm{a}, \mathrm{b}, *}$, A. Fernandes ${ }^{\mathrm{a}, \mathrm{b}}$ and J.-J. Sinou ${ }^{\mathrm{c}}$ \\ ${ }^{a}$ CNRS, UMR, Institut Jean Le Rond d'Alembert, Paris, France \\ ${ }^{\mathrm{b}}$ UPMC Université Paris, UMR, Institut Jean Le Rond d'Alembert, Paris, France \\ ${ }^{\mathrm{c}}$ Laboratoire de Tribologie et Dynamique des Systèmes UMR-CNRS, École Centrale de Lyon, Écully, France
}

Received 13 April 2012

Revised 9 November 2012

Accepted 14 January 2013

\begin{abstract}
The dynamics of a system and its safety can be considerably affected by the presence of cracks. Health monitoring strategies attract so a great deal of interest from industry. Cracks detection methods based on modal parameters variation are particularly efficient in the case of large cracks but are difficult to implement in the case of small cracks due to measurement difficulties in the case of small parameters variation. Therefore the present study proposes a new method to detect small cracks based on active modal damping and piezoelectric components. This method uses the active damping variation identificated with the Rational Fraction Polynomial algorithm as an indicator of cracks detection. The efficiency of the proposed method is demonstrated through numerical simulations corresponding to different crack depth and locations in the case of a finite element model of a clamped-clamped beam including four piezoelectric transducers.
\end{abstract}

Keywords: Crack detection, piezoelectric components, active damping, identification

\section{Introduction}

Cracks can considerably modify the dynamics of a system and affect system safety in several industrial applications. Crack modeling can be the first step of a health monitoring strategy. Cracks can be modelled using several approaches [14], for example reduction in element stiffness or pinned joint at crack location [19,26]. Rigid finite element method can be used to model cracks with a set of spring-damping elements of variable stiffness as shown by Kulesza and Sawicki [20]. The approach can be first linear if the crack is supposed to be opened [12] and second non linear if the crack breathing is taken into account $[2,9,24]$. Christides and Barr [10] shown that cracks can be modelled using exponential stiffness reduction in the case of Euler Bernoulli beams. This model takes into account the second moment of area reduction induced by the crack. Sinha et al. [31] proposed a simplified model for the location of cracks based on a triangular variation of the stiffness in beam structures using measured vibration data. This approach based on a local formulation of the flexibility permits particularly to include the crack model in a finite element model assuming that the stiffness reduction all fall within a single element.

One way to detect cracks on structures is to employ modal testing in which changes in modal parameters such as variations in frequencies and mode shapes are used to detect damage. A review based on the detection of structural damage through changes in frequencies was discussed by Salawu [28]. Moreover, Dilena and Morassi [11] proposed damage identification based on changes in the nodes of mode shapes. They demonstrated that appropriate use of

\footnotetext{
${ }^{*}$ Corresponding author: B. Chomette, Institut Jean Le Rond d'Alembert, UMR-CNRS 7190, CNRS, F-75005 Paris, France. E-mail: baptiste.chomette@upmc.fr.
} 
resonances and antiresonances can be used to avoid the non-uniqueness of damage location for symmetrical beams. Faverjon and Sinou [12] demonstrated that it is possible to detect the number of cracks in a beam and estimate both crack positions and sizes despite the presence of noise levels. Cracks can also be detected using observer [30] or using non linear phenomena in the case of cracked beams submitted to forced vibrations [1] or in the case of cracked rotors [29].

Piezoelectric components are usually used in health monitoring systems coupling to active control strategies such as active collocated control [23], active modal control [7,15] or passive strategies [4,17]. These strategies can be used in complex applications such as curved panels in a car body as shown by Hurlebaus [18], using evaluated modal parameters [32] or in the case of 3D mechanical structures [16]. There are more various finite element approaches to model piezoelectric components, using 3D-solid elements, plate or beam elements. Benjeddou [5] proposed a review of the different piezoelectric finite elements, solid, shell, plate and beam. Sadilek and Zemcik [27] proposed a one-dimensional beam element based on Euler-Bernoulli theory using a bilinear distribution of the electric field potential [13]. This model permits particularly to link one dimensional piezoelectric and cracks theory in order to develop health monitoring strategies.

In the case of controlled structures or smart-structures including actuators, sensors and control loop, cracks can considerably modify the dynamics of the controlled system. Chomette et al. [6] shown that active modal control based on Linear Quadratic Algorithm is highly sensitive to the variation of boundary conditions and thus to structure modification. They demonstrated that differences between the cracked structure and the modal model used in the controller based on the uncracked structure lead to control performance decrease. Chomette and Sinou [8] investigated the possibility of detecting transversal cracks in controlled truss structures using variation in the amplitude of the controlled system frequency response function and shown that the control performance variation can be an indicator of transversal crack detection.

Therefore the present study proposes a new method to detect small cracks based on piezoelectric components and active modal damping. Firstly the model of a cracked Euler-Bernoulli beam including piezoelectric elements is proposed. The main purpose of the control system is here to detect cracks. Consequently, the control algorithm is secondly designed in order to see small cracks. The novelty of the proposed strategy is to applied active control to monitor the structure behavior. Finally the efficiency of the proposed method is demonstrated through numerical simulations corresponding to different crack depths and locations in the case of the finite element model of a clamped-clamped beam including four piezoelectric transducers.

\section{Finite element modeling}

In this section the finite element modeling of a multi-cracked Euler Bernoulli beam including piezoelectric components is proposed. Firstly the crack modeling extended on several elements and the piezoelectric modeling are detailed. Secondly the finite element modeling of the complete structure including cracks and piezoelectric elements is proposed.

\subsection{Crack modeling}

Christides and Barr [10] shown that the effect of a crack in a continuous rectangular beam can be considered using an exponential variation of the flexural stiffness $E I$ where $E$ is the Young modulus and $I$ the second moment of area. This stiffness reduction can be approximated by a triangular reduction for the cracked element as explained by Sinha et al. [31] in order to include the reduction in a finite element model. If the stiffness reduction extends over more than one element the proposed approach have to be extended on several elements as shown in Fig. 1. Thus the stiffness reduction can be written in local coordinates for the $p^{\text {th }}$ element of length $\ell_{e}$

$$
E I^{ \pm}\left(x_{e}, p\right)=E I_{0} \pm \frac{E\left(I_{0}-I_{c}\right)}{\ell_{c}}\left(x_{e}+(p-1) \ell_{e}-x_{c}^{ \pm}\right),
$$

where $E, b, e_{p}$ and $I_{0}$ are the Young modulus, the weigth, the depth and the second moment of area of the uncracked beam respectively. $e_{c}$ and $I_{c}$ are the crack depth and the second moment of area of the cracked beam respectively. 


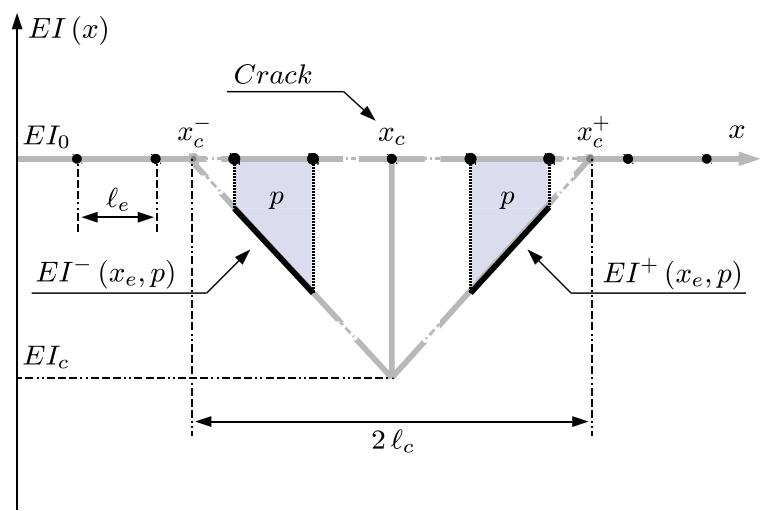

Fig. 1. Triangular stiffness reduction extended on several elements.

${ }^{+}$and ${ }^{-}$define the left and right part of the crack as shown in Fig. 1. $x_{c}, x_{c}^{+}$and $x_{c}^{-}$are the crack location and the affected stiffness location in global coordinates respectively. The affected stiffness zone is defined so that

$$
x_{c}^{+}-x_{c}^{-}=2 \ell_{c}
$$

where $\ell_{c}=1.5 e_{p}$ is the length of the affected stiffness based on experimental data [14]. The stiffness matrices are obtained using the standard integration based on the variation in flexural rigidity $E I^{ \pm}\left(x_{e}, p\right)$

$$
\mathbf{K}_{e, \text { crack }}^{ \pm}=\int_{0}^{\ell_{e}} E I^{ \pm}\left(x_{e}, p\right) \mathbf{N}_{e}^{\prime \prime T}\left(x_{e}\right) \mathbf{N}_{e}^{\prime \prime}\left(x_{e}\right) d x_{e}
$$

where the shape functions $\mathbf{N}_{e}$ are those for a standard Euler-Bernoulli element beam. Finally, the element stiffness matrix including a crack can be written using the $p$ parameter

$$
\mathbf{K}_{e, \text { crack }}^{ \pm}=\mathbf{K}_{e}-\mathbf{K} c_{e}^{ \pm}(p)
$$

with

$$
\mathbf{K} c_{e}^{ \pm}(p)= \pm \frac{E\left(I_{0}-I_{c}\right)}{\ell_{c}}\left(\begin{array}{cccc}
\frac{6\left(2 X_{c}+\ell_{e}\right)}{\ell_{e}^{3}} & \frac{2\left(3 X_{c}+2 \ell_{e}\right)}{\ell_{e}^{2}} & -\frac{6\left(2 X_{c}+\ell_{e}\right)}{\ell_{e}^{3}} & \frac{2\left(3 X_{c}+\ell_{e}\right)}{\ell_{e}^{2}} \\
\frac{2\left(3 X_{c}+2 \ell_{e}\right)}{\ell_{e}^{2}} & \frac{\left(4 X_{c}+3 \ell_{e}\right)}{\ell_{e}} & -\frac{2\left(3 X_{c}+2 \ell_{e}\right)}{\ell_{e}^{2}} & \frac{\left(2 X_{c}+\ell_{e}\right)}{\ell_{e}} \\
-\frac{6\left(2 X_{c}+\ell_{e}\right)}{\ell_{e}^{3}} & -\frac{2\left(3 X_{c}+2 \ell_{e}\right)}{\ell_{e}^{2}} & \frac{6\left(2 X_{c}+\ell_{e}\right)}{\ell_{e}^{3}} & -\frac{2\left(3 X_{c}+\ell_{e}\right)}{\ell_{e}^{2}} \\
\frac{2\left(3 X_{c}+\ell_{e}\right)}{\ell_{e}^{2}} & \frac{\left(2 X_{c}+\ell_{e}\right)}{\ell_{e}} & -\frac{2\left(3 X_{c}+\ell_{e}\right)}{\ell_{e}^{2}} & \frac{\left(4 X_{c}+\ell_{e}\right)}{\ell_{e}}
\end{array}\right)
$$

where $X_{c}=x_{c}^{ \pm}-p \ell_{e}$ and $-\mathbf{K} c_{e}^{ \pm}(p)$ is the stiffness reduction induced by the crack on the $p^{\text {th }}$ element and $\mathbf{K}_{e}$ the element stiffness matrix of an uncracked element.

Using this modeling, crack location is independant of the mesh and the mesh size can be modified to take into account other physical coupling like electromechanical coupling using piezoelectric elements. Due to the detection method based on linear control, the crack is supposed to be open and the breathing crack is not taken into account.

\subsection{Piezoelectric finite element modeling}

The finite element modeling of a structure including piezoelectric elements $[5,22,27]$ can be written

$$
\left\{\begin{array}{l}
\mathbf{M}_{u u} \ddot{\mathbf{u}}+\mathbf{K}_{u u}^{c c} \mathbf{u}+\mathbf{K}_{u \phi} \boldsymbol{\phi}=\mathbf{F} \\
\mathbf{K}_{\phi u} \mathbf{u}+\mathbf{K}_{\phi \phi} \boldsymbol{\phi}=\mathbf{Q}
\end{array},\right.
$$


where $\mathbf{M}_{u u}, \mathbf{K}_{u u}^{c c}, \mathbf{K}_{u \phi}=\mathbf{K}_{\phi u}^{T}$ and $\mathbf{K}_{\phi \phi}$ are the mass, the short-circuit stiffness, the electromechanical coupling and the dielectric matrix respectively. $\mathbf{u}, \phi, \mathbf{F}$ and $\mathbf{Q}$ are the mechanical displacement vector and electrical potential, the force and the electrical charge vector respectively. The generalized electromechanical coupling coefficient $k_{\ell}$ is defined [17] for the $\ell^{\text {th }}$ mode as

$$
k_{\ell}^{2}=\frac{\omega_{c o \ell}^{2}-\omega_{c c \ell}^{2}}{\omega_{c o \ell}^{2}},
$$

where $\omega_{c o_{\ell}}$ and $\omega_{c c_{\ell}}$ are the $\ell^{\text {th }}$ pulsations with open and short circuited electrodes respectively. The generalized electromechanical coupling coefficient is used to characterize piezoelectric actuators and sensors efficiency in the control algorithm.

\subsection{Model of the complete beam}

The finite element model of the complete beam including multi-cracks and piezoelectric elements permits to take into account cracks in the generalized electromechanical coupling coefficient. It can be written using Eqs (4) and (6)

$$
\left\{\begin{array}{l}
\mathbf{M}_{u u} \ddot{\mathbf{u}}+\left(\mathbf{K}_{u u}^{c c}-\mathbf{K}_{c r a c k}\right) \mathbf{u}+K_{u \phi} \phi=\mathbf{F} \\
\mathbf{K}_{\phi u} \mathbf{u}+\mathbf{K}_{\phi \phi} \phi=\mathbf{Q}
\end{array}\right.
$$

where $\mathbf{K}_{\text {crack }}$ is the crack stiffness matrix after assembling the different beam elements of stiffness $\mathbf{K}_{e, \text { crack }}^{ \pm}$.

\section{Modal control}

Modal control is usually applied to reduce structural vibration in targeting the control energy only on the modes of interest and permits to reduce the actuators and sensors number [15]. The control algorithm is designed here in order to detect small cracks. The Linear Quadratic Gaussian (LQG) algorithm is chosen in this application to target the control only on the mode of interest with the weighting matrix in order to see the modal influence of the cracks. The LQG algorithm is based on a linear model of the structure.

\subsection{Linear system}

The linear control [23] is based on a linear model which can be described in the state form by

$$
\left\{\begin{array}{l}
\dot{\mathbf{x}}=\mathbf{A x}+\mathbf{B u}^{*}+\mathbf{W} w \\
\mathbf{y}=\mathbf{C x}
\end{array}, \mathbf{u}^{*}=-\mathbf{G} \hat{\mathbf{x}},\right.
$$

where $\mathbf{A}$ is the dynamical system matrix, $\mathbf{B}$ and $\mathbf{C}$ are the actuator and sensor matrices respectively and $\mathbf{W}$ is the excitation matrix. $\mathbf{x}$ is the state vector in modal coordinates. $\mathbf{y}={ }^{t}\left(\begin{array}{ll}y_{1} & y_{2}\end{array}\right)$ is the output vector detailed here in the case of two sensors. $\mathbf{u}^{*}={ }^{t}\left(\begin{array}{ll}u_{1}^{*} & u_{2}^{*}\end{array}\right)$ is the optimal control solution of the Linear Quadratic Regulator problem, detailed here in the case of two actuators and calculated using the estimated state $\hat{\mathbf{x}}$ and the gain matrix $\mathbf{G} . w$ is the excitation applied on the beam and choosen to be unitary in this study. In practical applications, a white noise excitation can be applied using the piezoelectric actuators. The state matrices can be written for $n$ modes, 2 actuators and 2 sensors

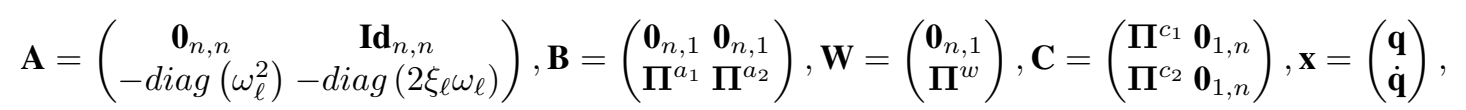


with $\ell=\{1, n\}$, where $\mathbf{q}$ is the modal displacement vector, $\omega_{\ell}$ and $\xi_{\ell}$ are the natural frequencies and damping respectively. $\Pi^{a_{i}}, \Pi^{c_{i}}$ and $\Pi^{w}$ are the $i^{\text {th }}$ actuator, $i^{\text {th }}$ sensor and disturbance vectors in the modal basis respectively $(i=\{1,2\})$. In the case of piezoelectric actuators and sensors, the $\ell^{\text {th }}$ electromechanical coupling vector composants corresponding to the $i^{\text {th }}$ actuator can be written [6]

$$
\left(\Pi_{\ell}^{a_{i}}\right)^{2}=\frac{\left(k_{\ell}^{a_{i}}\right)^{2} C_{p}^{a_{i}}\left(\omega_{c c_{\ell}}\right)^{2}}{1-\left(k_{\ell}^{a_{i}}\right)^{2}}
$$

where ${ }^{a}$ indicates actuator. $k$ is the electromechanical coupling coefficient and $C_{p}$ the piezoelectric component capacity. The piezoelectric component whose electromechanical coupling coefficient $k$ is calculated using the Eq. (7), is modelled with open electrodes and all the other components are modelled with short circuited electrodes so that the only piezoelectric effect is due to the considered component.

\subsection{Transfer functions in open and closed loop}

The transfer function of the uncontrolled (open-loop) system $\mathbf{H}_{o l}$ can be written using Eq. (9) for one sensor of response $y_{1}$ as

$$
\mathbf{H}_{o l}(j \omega)=\frac{y_{1}}{w}=\mathbf{C}_{s}\left(j \omega \mathbf{I d}-\mathbf{A}_{s}\right)^{-1} \mathbf{W}_{s}
$$

where $\left(\mathbf{A}_{s}, \mathbf{B}_{s}, \mathbf{C}_{s}, \mathbf{W}_{s}\right)$ is the state model that represents the structure in the numerical simulations. Frequencies and damping variation induced by the cracks are taken into account in the matrix $\mathbf{A}_{s}$. Mode shapes and electromechanical coupling coefficient variation induced by the cracks are taken into account in the $\mathbf{B}_{s}, \mathbf{C}_{s}$ and $\mathbf{W}_{s}$ matrices. The transfer function of the system controlled (closed-loop) $\mathbf{H}_{c l}$ can be written for one sensor as

$$
\mathbf{H}_{c l}(j \omega)=\frac{y_{1}}{w}=\mathbf{C}_{s}\left(j \omega \mathbf{I d}-\left(\mathbf{A}_{s}-\mathbf{B}_{s} \mathbf{G}\left(j \omega \mathbf{I d}-\left(\mathbf{A}_{m}-\mathbf{B}_{m} \mathbf{G}-\mathbf{L} \mathbf{C}_{m}\right)\right)^{-1} \mathbf{L C}_{s}\right)\right)^{-1} \mathbf{W}_{s}
$$

where $\left(\mathbf{A}_{m}, \mathbf{B}_{m}, \mathbf{C}_{m}\right)$ is the state model used by the Luenberger observer [21] to evaluate the state vector. $\mathbf{G}$ and $\mathbf{L}$ are the Linear Quadratic gain (LQ) and the observer gain of the Linear Quadratic Gaussian algorithm (LQG) [3] respectively.

\subsection{Gain calculation to detect cracks}

In the case of small cracks the frequency shift induced by the stiffness reduction cannot be easily measured. The proposed method consists in studying the dynamic of the closed loop system and to modify the control gain $\mathbf{G}$ in order to detect the cracks. The gain $\mathbf{G}$ is obtained using the quadratic criterion [23]

$$
J=\int_{0}^{\infty} \mathbf{x}(t)^{T} \mathbf{Q} \mathbf{x}(t) d t
$$

where $\mathbf{Q}$ is the classical weighting matrix. It can be written for $n$ modes

$$
\mathbf{Q}=\left(\begin{array}{ll}
\mathbf{Q}_{\text {sup }} & \mathbf{0}_{n, n} \\
\mathbf{0}_{n, n} & \mathbf{Q}_{i n f}
\end{array}\right)
$$

The principle of the method is to design a little robust controller highly sensitive to the structure modifications. Therefore, the controller performance must be high and the robustness low. In this study, only the even modes are weighted using the $\mathbf{Q}$ matrix so that a frequency shift and an electromechanical coupling coefficient variation induce a slip of the controller effects on the even modes. In the case of uncracked structures only the weighted modes are highly actively damped using $\mathbf{Q}_{\text {sup }}$ and $\mathbf{Q}_{\text {inf }}$ and there is no effect on the odd modes. Weighting coefficient are arbitrarily chosen to target the control only on the wanted modes. In this study, three ponderations $\left(\mathbf{Q}_{1}, \mathbf{Q}_{2}\right.$ and $\left.\mathbf{Q}_{3}\right)$ of the LQG controller are tested and the maximal weighting matrix $\mathbf{Q}_{3}$ is defined so that $\mathbf{Q}_{\text {sup }}=\operatorname{diag}\left(\begin{array}{lllllllllll}1 & 1 \text { e } 15 & 1 & 1 & 17 & 1\end{array}\right)$ and $\mathbf{Q}_{\text {inf }}=\operatorname{diag}\left(\begin{array}{lllll}1 & 1 & 1 & 1 & 1\end{array}\right)$. In the case of cracked structures, the structural modifications induced by the cracks modify the controller dynamics and induce some active damping on the odd modes whereas the damping of the even modes decreases. 
Table 1

Elastic, piezoelectric and dielectric constants of piezoelectric materials

\begin{tabular}{cccccccc}
\hline$c_{11}^{E}(\mathrm{GPa})$ & $c_{22}^{E}$ & $c_{33}^{E}$ & $c_{12}^{E}$ & $c_{13}^{E}$ & $e_{31}\left(\mathrm{C} / \mathrm{m}^{2}\right)$ & $e_{33}$ & $\epsilon_{33}^{S}(\mathrm{nF} / \mathrm{m})$ \\
\hline 127.2 & 127.2 & 117.4 & 802.1 & 846.7 & -6.6 & 23.2 & 8.85 \\
\hline
\end{tabular}

Table 2

Modal parameters of the uncracked uncontrolled beam

\begin{tabular}{ccc}
\hline Mode & Frequency $[\mathrm{Hz}]$ & Damping \\
\hline 1 & 427.8 & $5.00 \times 10^{-4}$ \\
2 & 1148.5 & $5.00 \times 10^{-4}$ \\
3 & 2205.5 & $7.70 \times 10^{-4}$ \\
4 & 3590.4 & $1.18 \times 10^{-3}$ \\
\hline
\end{tabular}

Case 1:

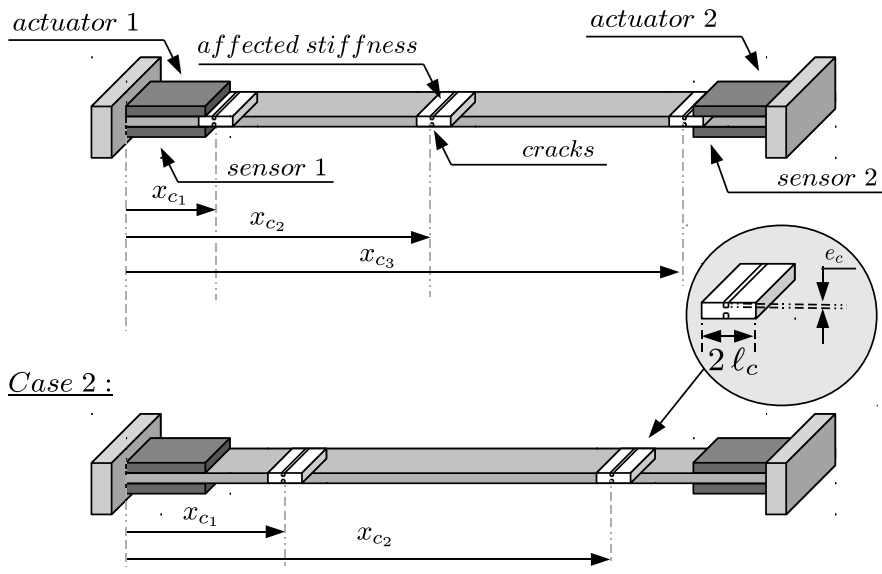

Fig. 2. Multi-cracked composite beam in case 1 and 2.

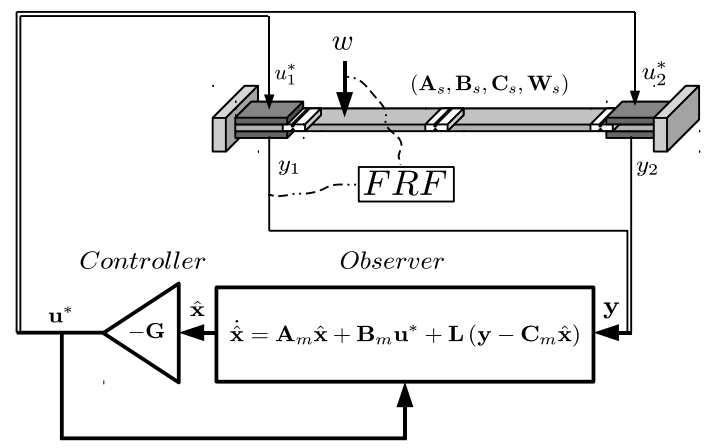

Fig. 3. Controlled multi-cracked composite beam.

\section{Numerical results}

The clamped-clamped beam studied (length $L_{p}=200 \mathrm{~mm}$, thickness $e_{p}=3 \mathrm{~mm}$ and width $b_{p}=20 \mathrm{~mm}$ ) presented in Fig. 2 includes two piezoelectric actuators and two piezoelectric colocated sensors (length $L_{a s}=$ $20 \mathrm{~mm}$, thickness $e_{a s}=0.2 \mathrm{~mm}$ and width $b_{a s}=20 \mathrm{~mm}$ ) located closed to the both clamping and made in PZT-5H whose mechanical and electromechanical properties can be found in Table 1.

Numerical simulations of Section 4 are based on the open (without control) and closed (with control) loop transfer function between the excitation and the first piezoelectric sensor of the cracked structure. Modal parameters of the uncracked uncontrolled beam identified using the Rational Fraction Polynomial (RFP) algorithm [25] applied to the Frequency Response Function of the finite element model are detailed in Table 2.

Two crack location cases are studied to demonstrate the robustness of the detection method. In the first case three cracks are located on $x_{c_{1}}=2.4 \mathrm{~mm}, x_{c_{2}}=1 \mathrm{~mm}$ and $x_{c_{3}}=17.6 \mathrm{~mm}$. In the second case two cracks are located at $x_{c_{1}}=6 \mathrm{~mm}$ and $x_{c_{2}}=14 \mathrm{~mm}$. The crack depth $\mu=\frac{e_{c}}{e_{p}}$ varies from 0 to $\frac{1}{4}$.

The control loop presented in Fig. 3 includes a LQG controller based on a Luenberger observer. Frequency Response Functions (FRF) are measured between the excitation $w$ and the first piezoelectric sensor. $\mathbf{u}^{*}$ is the optimal control calculated using the LQ gain $\mathbf{G}, u_{1}^{*}$ and $u_{2}^{*}$ are the control composants applied on the actuators 1 and 2 . $\mathbf{y}$ is the output vector where $y_{1}$ and $y_{2}$ are the composants of the sensors 1 and 2 . The model $\left(\mathbf{A}_{s}, \mathbf{B}_{s}, \mathbf{C}_{s}, \mathbf{W}_{s}\right)$ represents the multi-cracked beam including ten modes and $\left(\mathbf{A}_{m}, \mathbf{B}_{m}, \mathbf{C}_{m}\right)$ is the model of the uncracked structure including five modes used by the Luenberger observer to reconstruct the state vector $\hat{\mathbf{x}}$.

The study focusses on the first fourth modes. Three ponderations $\left(\mathbf{Q}_{1}, \mathbf{Q}_{2}\right.$ and $\left.\mathbf{Q}_{3}\right)$ of the LQG controller are tested in the two cases in order to show the proposed method efficiency, with $\mathbf{Q}_{3} \gg \mathbf{Q}_{2} \gg \mathbf{Q}_{1}$.

\subsection{First case: Three cracks}

In this first case three cracks are located on $x_{c_{1}}=2.4 \mathrm{~mm}, x_{c_{2}}=1 \mathrm{~mm}$ and $x_{c_{3}}=17.6 \mathrm{~mm}$. A first study is carried out to show the influence of the crack depth on the electromechanical coupling coefficient. Indeed, the 


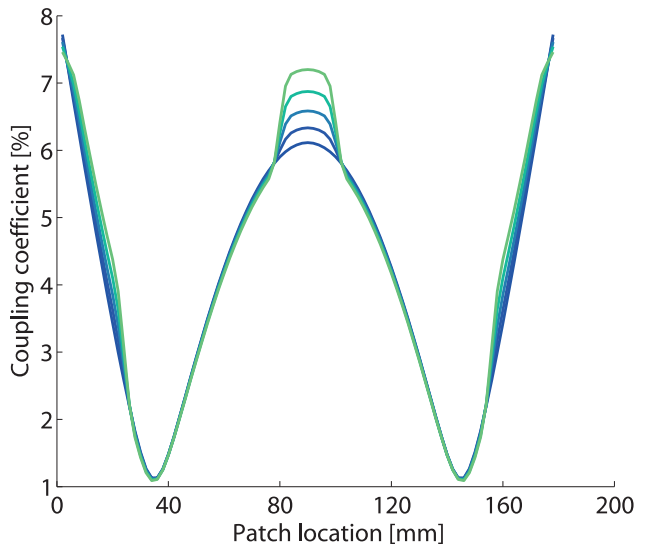

(a) First mode

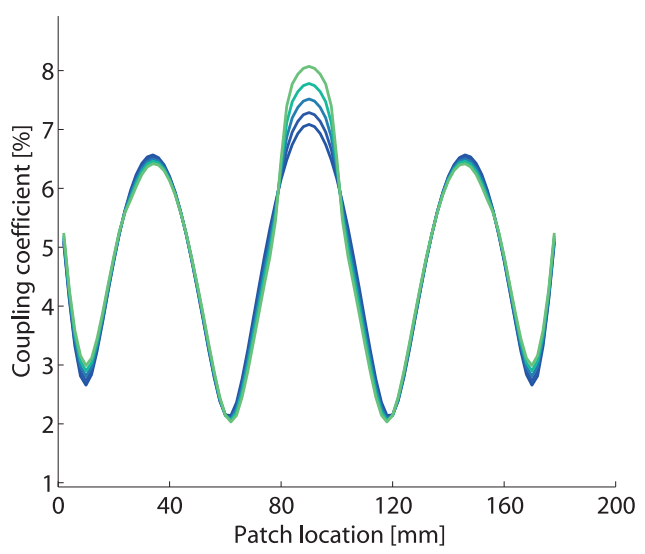

(c) Third mode

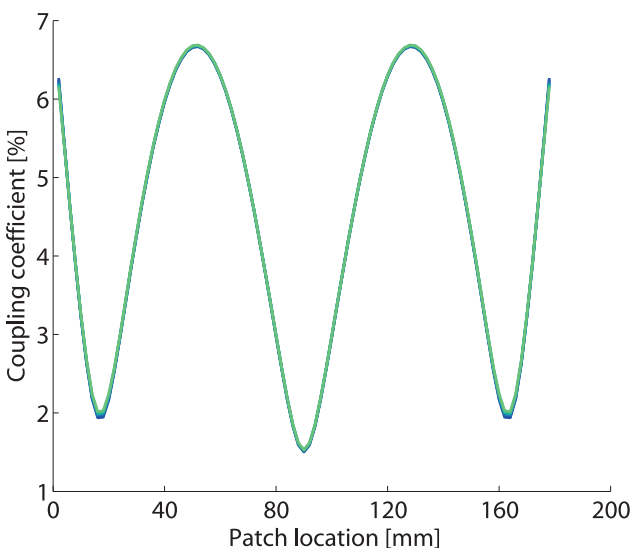

(b) Second mode

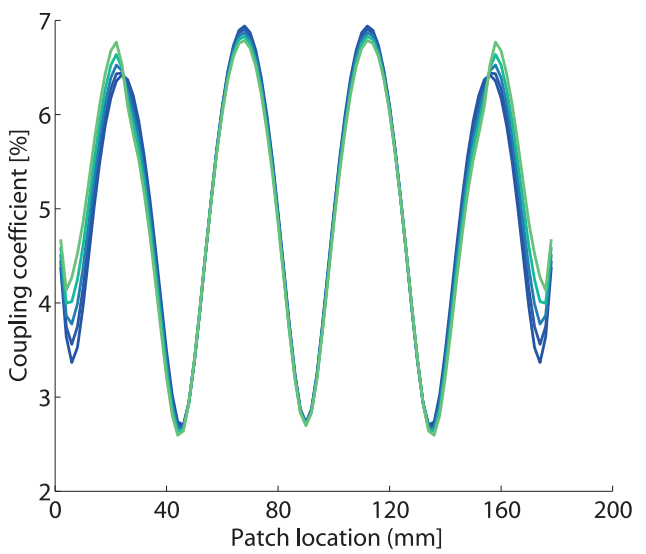

(d) Fourth mode

Fig. 4. Electromechanical coupling coefficient for one piezoelectric patch on the beam from $x_{a s}=0 \mathrm{~mm}$ to $x_{a s}=180 \mathrm{~mm}$ and for a crack depth from $\mu=0$ to $\mu=1 / 4$ (ם: $\mu=0$, $\mathbf{\square}: \mu=1 / 16$, $\mathbf{\square}: \mu=1 / 8, \square: \mu=3 / 16, \square: \mu=1 / 4)$.

piezoelectric actuators are here located in the section where the stiffness is affected by the first and fourth crack. This first study permits to know if the electromechanical coupling coefficient of the actuators and consequently the $\mathbf{B}_{s}$ and $\mathbf{C}_{s}$ matrices of the controller are affected or not. The coupling coefficient is calculated for several locations of one piezoelectric patch on the beam from $x_{a s}=0 \mathrm{~mm}$ to $x_{a s}=180 \mathrm{~mm}$, where $x_{a s}$ defines the left corner coordinate of the patch, and for a crack depth from $\mu=0$ to $\mu=1 / 4$. The evolution of the coupling coefficient is presented in Figs 4(a) to (d). In these figures, the location of the piezoelectric component changes along the beam to look into all the sections of the structure where the stiffness is affected by the cracks. The excitation application point is constant. In the data shown in Figs 5 to 8, the piezoelectric components and the force vector location are constants. There is a large variation of the coupling coefficient for the first and third mode when the patch is above the second crack. There is a large variation of the coupling coefficient for the fourth mode when the patch is above the first or third crack. There is a very small modification for the second mode. These modifications will be taken into account in the closed loop system dynamics calculation particularly in the $\mathbf{B}_{s}$ and $\mathbf{C}_{s}$ matrices which include the coupling coefficient. In closed loop, frequency and electromechanical coupling coefficient shift induce some differences between the state model of the controller and the real structure $\left(\mathbf{A}_{s} \neq \mathbf{A}_{m}, \mathbf{B}_{s} \neq \mathbf{B}_{m}\right.$ and $\mathbf{C}_{s} \neq \mathbf{C}_{m}$ ). These differences can modify the closed loop structure dynamics and consequently induce some performance variations.

Frequency response functions of the uncontrolled and controlled beam for three ponderations $\mathbf{Q}_{1}, \mathbf{Q}_{2}$ and $\mathbf{Q}_{3}$ are shown in Figs 5(a)-(c) for the first mode, in Figs 5(d)-(f) for the second mode, in Figs 5(g)-(i) for the third mode and in Figs 5(j)-(1) for the fourth mode. It can be observed that the presence of cracks decrease the frequencies of each mode. Moreover, increasing the crack size decrease modal frequencies. For the uncontrolled beam, the frequency 


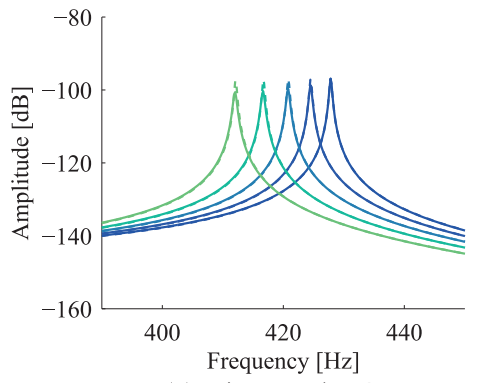

(a) First mode, $\mathbf{Q}_{1}$

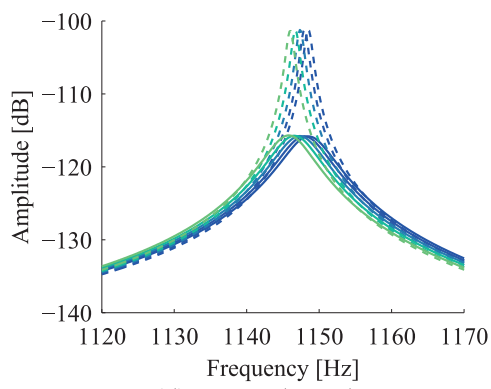

(d) Second mode, $\mathbf{Q}_{1}$

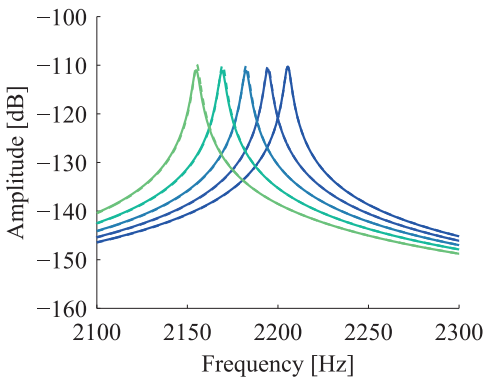

(g) Third mode, $\mathbf{Q}_{1}$

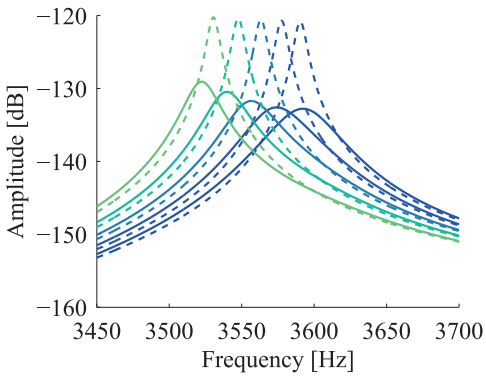

(j) Fourth mode, $\mathbf{Q}_{1}$

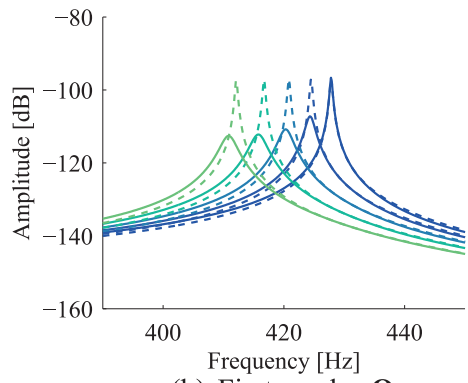

(b) First mode, $\mathbf{Q}_{2}$

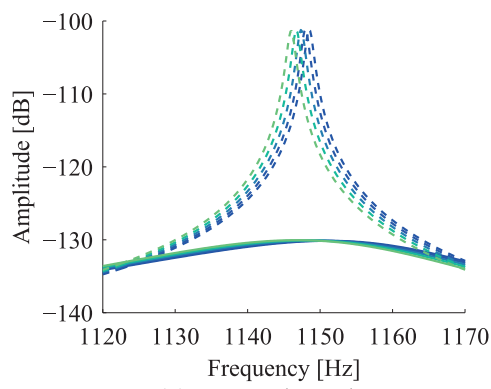

(e) Second mode, $\mathbf{Q}_{2}$

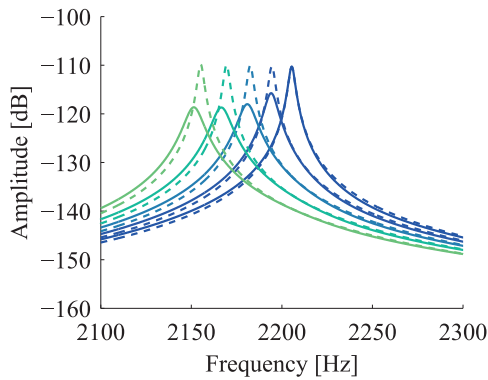

(h) Third mode, $\mathbf{Q}_{2}$

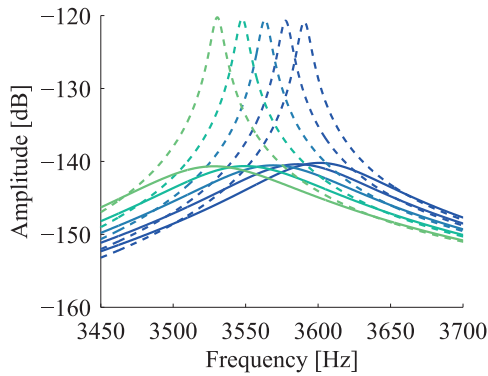

(k) Fourth mode, $\mathbf{Q}_{2}$

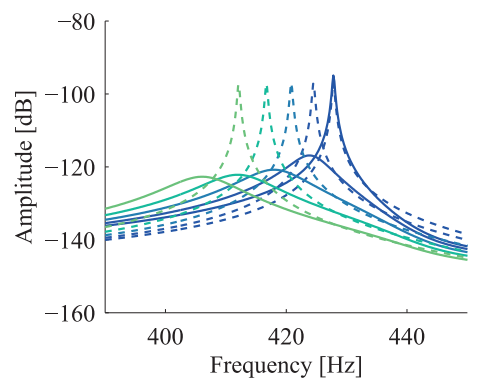

(c) First mode, $\mathbf{Q}_{3}$

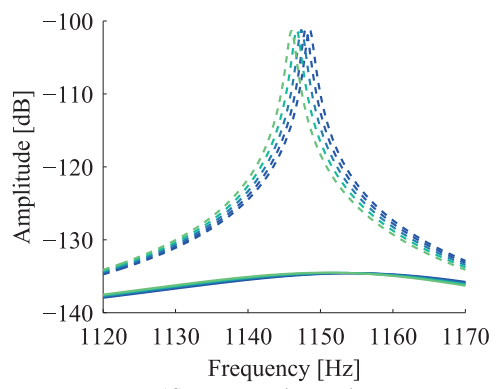

(f) Second mode, $\mathbf{Q}_{3}$

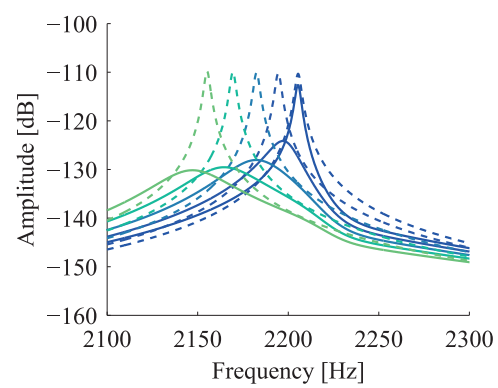

(i) Third mode, $\mathbf{Q}_{3}$

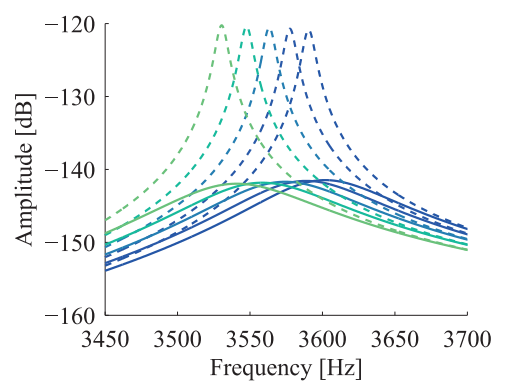

(1) Fourth mode, $\mathbf{Q}_{3}$

Fig. 5. Uncontrolled (- -) and controlled (-) FRF of the multi-cracked beam in the first case for the first fourth mode ( $\square: \mu=0$, $\square: \mu=1 / 16$, $\square: \mu=1 / 8, \square: \mu=3 / 16, \square: \mu=1 / 4)$ for three ponderations $\mathbf{Q}_{1}, \mathbf{Q}_{2}$ and $\mathbf{Q}_{3}$.

variation induced by the crack depth variation from $\mu=1 / 16$ to $\mu=1 / 4$, usually measured to detect cracks, is about -0.8 to $-3.8 \%$ for the first mode, 0 to $-0.2 \%$ for the second mode, -0.5 to $-2.3 \%$ for the third mode and -0.4 to $-1.7 \%$ for the fourth mode. Howeover, due to the small depth of each cracks, detection of the presence of cracks based on the evolution of natural frequencies appears to be not efficient. Identified frequency and damping for three weighting matrices are presented in Figs 6(a)-(d) for the first fourth modes. The modal structural damping variation in less than $1 \%$ for the four modes. The damping variation for the controlled beam using the $\mathbf{Q}_{1}$ weighting matrix is about 18.6 to $33.8 \%$ for the first mode and 7.6 to $12.6 \%$ for the third mode, 72 to $85 \%$ for the first mode 

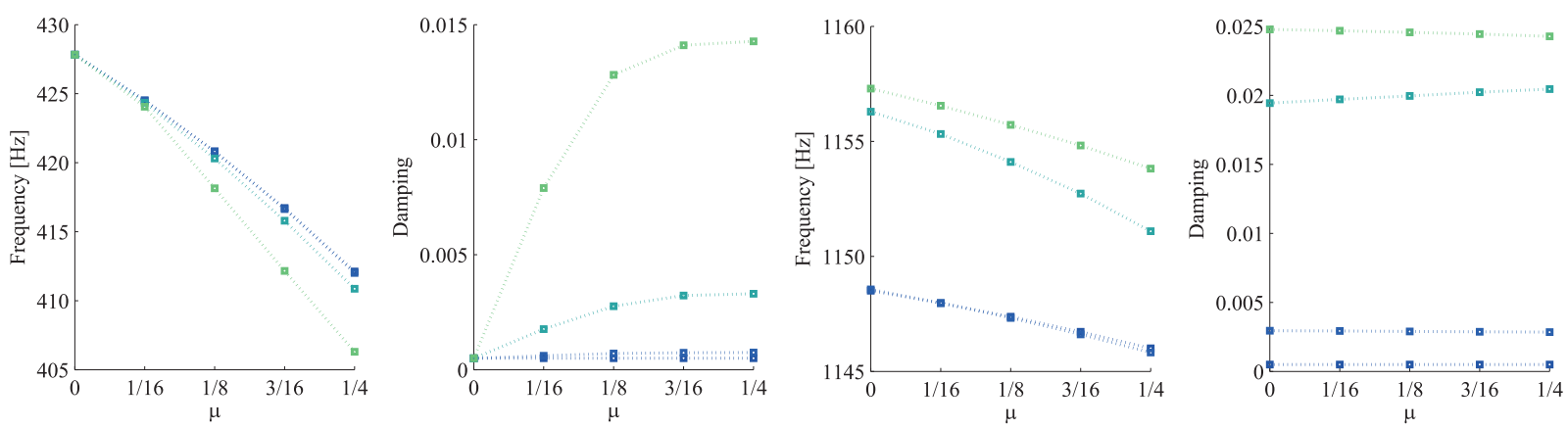

(a) First mode
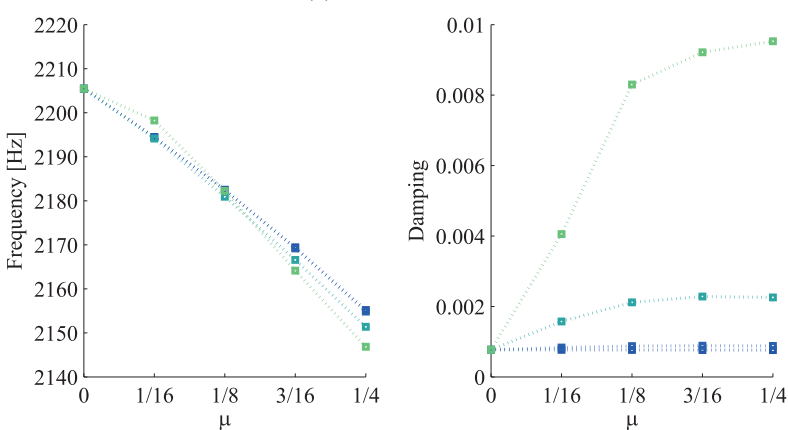

(c) Third mode
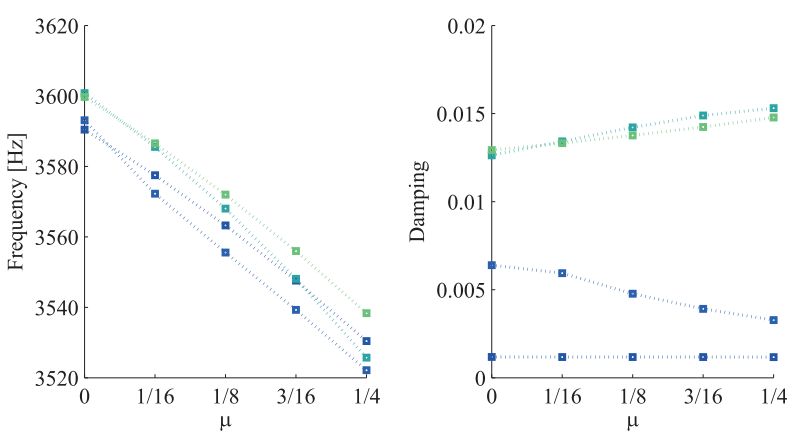

(d) Fourth mode

Fig. 6. Frequency and damping in the first case for three weighting matrices ( $\mathbf{\square}$ : uncontrolled, $\left.\mathbf{\square}: \mathbf{Q}_{1}, \mathbf{\square}: \mathbf{Q}_{2}, \mathbf{\square}: \mathbf{Q}_{3}\right)$

and 51.1 to $65.9 \%$ for the third mode using the $\mathbf{Q}_{2}$ weighting matrix, 93.7 to $96.5 \%$ for the first mode and 81.0 to $91.9 \%$ for the third mode using the $\mathbf{Q}_{3}$ weighting matrix.

\subsection{Second case: Two cracks}

In this second case two cracks are located at $x_{c_{1}}=6 \mathrm{~mm}$ and $x_{c_{2}}=14 \mathrm{~mm}$. Frequency response functions of the uncontrolled and controlled beam for three ponderations $\mathbf{Q}_{1}, \mathbf{Q}_{2}$ and $\mathbf{Q}_{3}$ are shown in Figs 7(a)-(c) for the first mode, in Figs 7(d)-(f) for the second mode, in the Figs 7(g)-(i) for the third mode and in Figs 7(j)-(1) for the fourth mode. There is the same decrease of the frequencies induced by the cracks depend on the crack depth. For the uncontrolled beam, the frequency variation induced by the crack depth variation from $\mu=1 / 16$ to $\mu=1 / 4$, usually measured to detect cracks, is about -0.1 to $-0.5 \%$ for the first mode, -0.8 to $-3.6 \%$ for the second mode, -0.3 to $-1.4 \%$ for the third mode and -0.1 to $-0.4 \%$ for the fourth mode. Consequently, the evolution of natural frequencies appears to be not efficient to detect cracks. Identified frequency and damping for three weighting matrices are presented in Figs 8(a)-(d) for the first fourth modes. The modal structural damping variation in less than $2 \%$ for the four modes. The damping variation for the controlled beam using the $\mathbf{Q}_{1}$ weighting matrix is about 5.0 to $13.8 \%$ for the first mode and 5.2 to $13.5 \%$ for the third mode, 28.9 to $64.7 \%$ for the first mode and 38.6 to $64.3 \%$ for the third mode using the $\mathbf{Q}_{2}$ weighting matrix, 66.1 to $90.9 \%$ for the first mode and 68.7 to $90.7 \%$ for the third mode using the $\mathbf{Q}_{3}$ weighting matrix.

The index variation is summarized in Fig. 9. The detection index based on frequency shift is calculated using the formula $100 \times \frac{f_{\text {cracked }}-f_{\text {uncracked }}}{f_{\text {cracked }}}$ and based on active modal damping using $100 \times \frac{\xi_{\text {cracked }}-\xi_{\text {uncracked }}}{\xi_{\text {cracked }}}$. Where $\xi$ denotes the identified total damping, that is to say the structural damping and the active damping whose variation is the most important. In the two cases, cracks induce a small frequency variation that cannot be experimentally measured and a very light structural damping variation. Using active damping, the damping variation is highly increased proportionally to the crack depth and induces a large amplitude variation of the controlled beam frequency response function that can be easily measured. The control is initially targeted on the second and third modes. The modal parameters variation, frequency shift, modal damping and mode shapes variation, induce some differences 


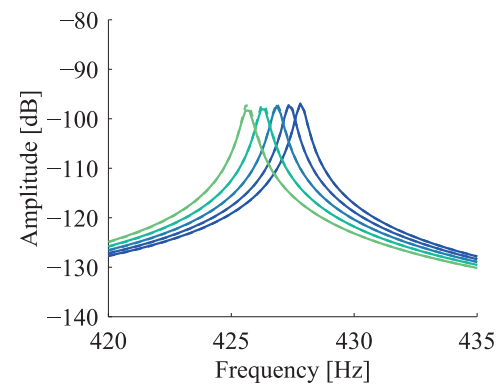

(a) First mode, $\mathbf{Q}_{1}$

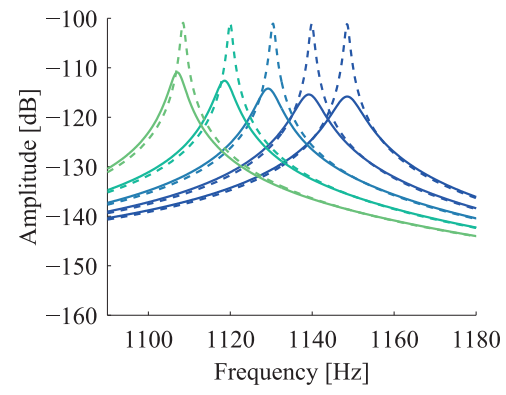

(d) Second mode, $\mathbf{Q}_{1}$

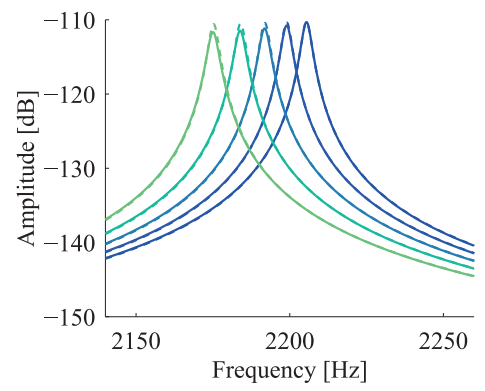

(g) Third mode, $\mathbf{Q}_{1}$

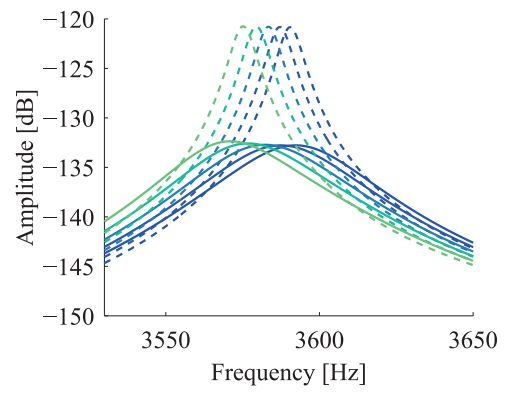

(j) Fourth mode $\mathbf{Q}_{1}$

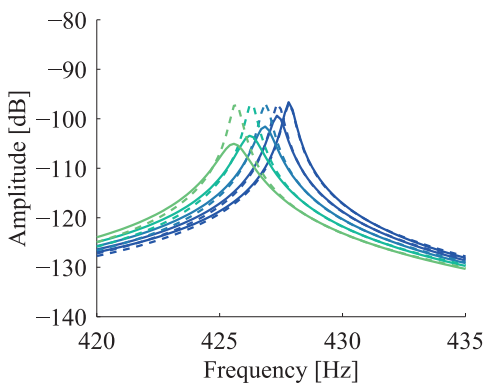

(b) First mode, $\mathbf{Q}_{2}$

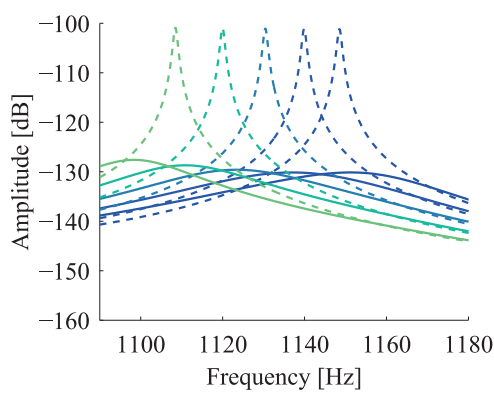

(e) Second mode, $\mathbf{Q}_{2}$

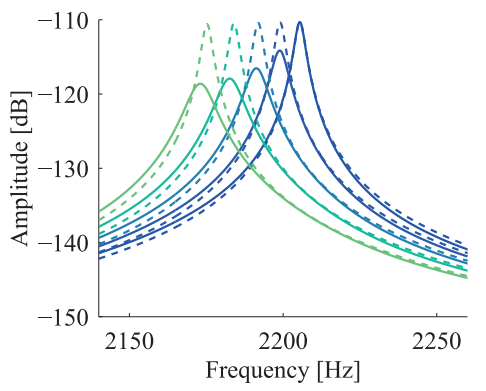

(h) Third mode, $\mathbf{Q}_{2}$

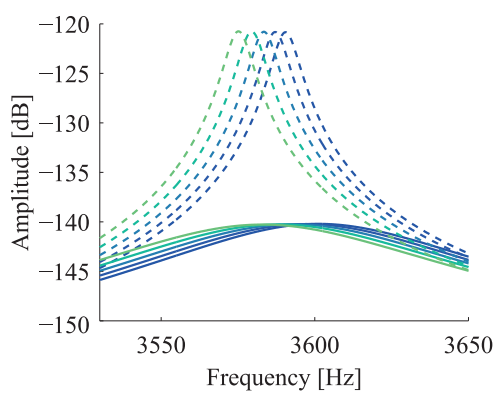

(k) Fourth mode $\mathbf{Q}_{2}$

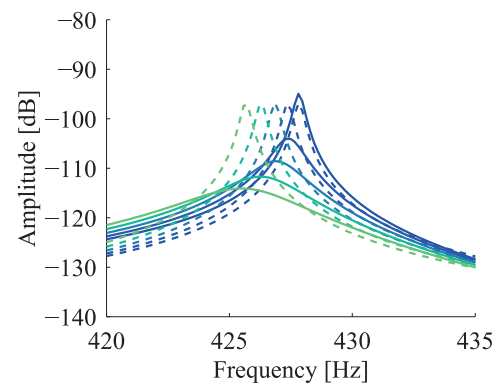

(c) First mode, $\mathbf{Q}_{3}$

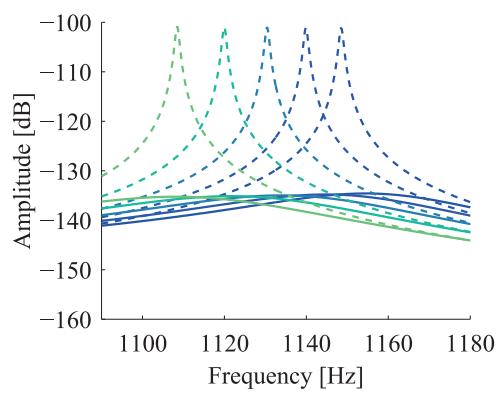

(f) Second mode, $\mathbf{Q}_{3}$

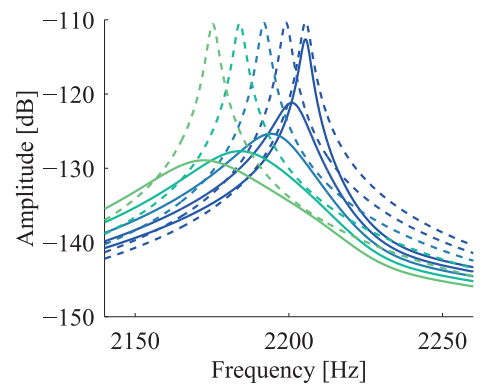

(i) Third mode, $\mathbf{Q}_{3}$

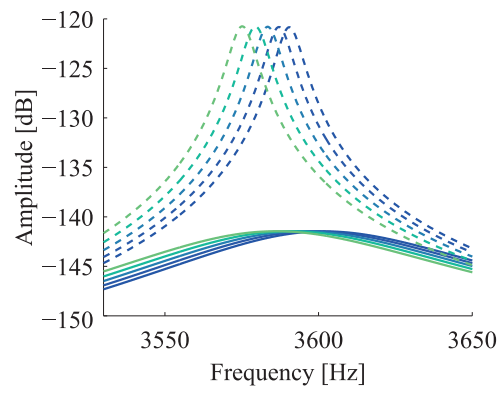

(1) Fourth mode $\mathbf{Q}_{3}$

Fig. 7. Uncontrolled (- -) and controlled (-) FRF of the multi-cracked beam in the second case for the first fourth mode ( $\square: \mu=0$, $\square: \mu=1 / 16$,

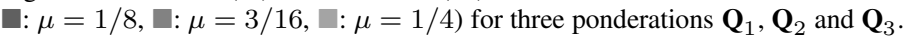

between the state model used by the controller and the real structure. These changes modify the controller behaviour and induce some active damping on the first and third modes initially very slightly controlled and are here used as an indicator of cracks presence. These results can be physically interpreted. The stiffness of the cracked structure is lower than the uncracked ones. The piezoelectric components are therefore more efficient and the electromechanical coupling coefficient is increased. This variation is taken into account in the $\mathbf{B}_{s}$ and $\mathbf{C}_{s}$ matrices. Moreover, the 


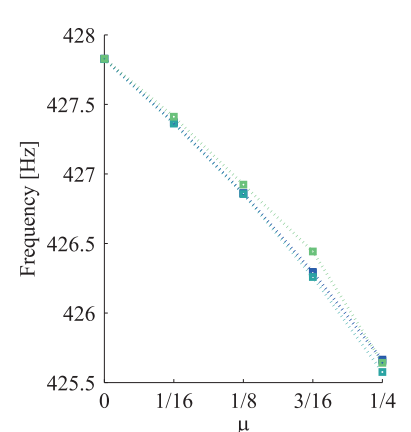

(a) First mode
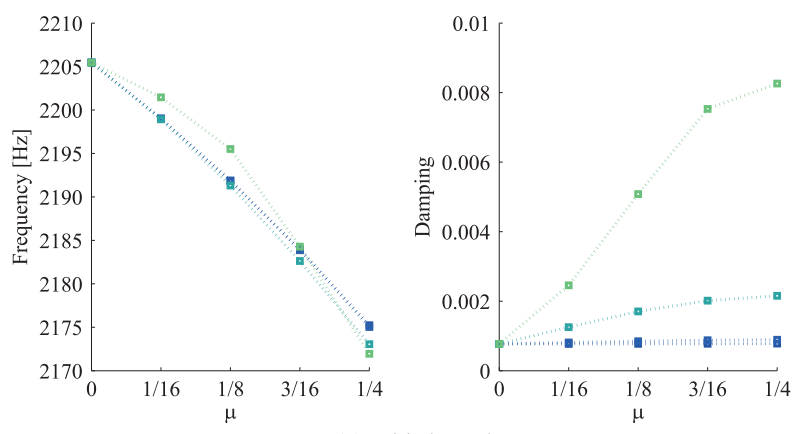

(c) Third mode

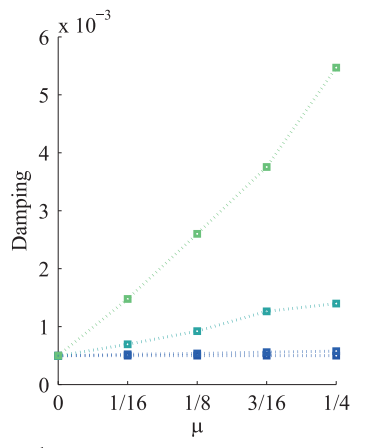

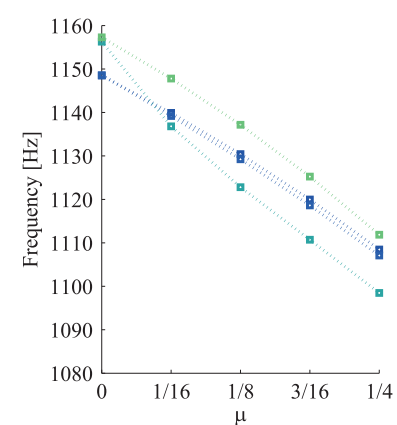

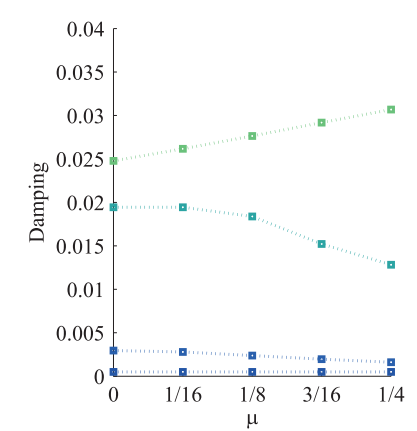

(b) Second mode
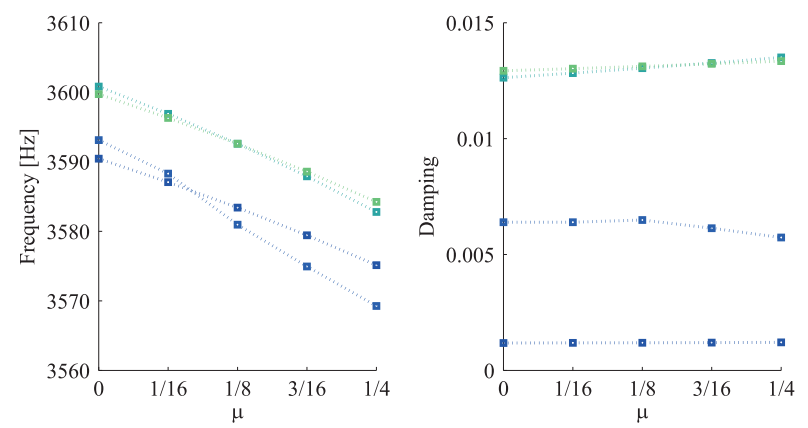

(d) Fourth mode

Fig. 8. Frequency and damping in the second case for three weighting matrices ( $\mathbf{\square}$ : uncontrolled, $\mathbf{\square}: \mathbf{Q}_{1}, \mathbf{\square}: \mathbf{Q}_{2}, \mathbf{\square}: \mathbf{Q}_{3}$ ).
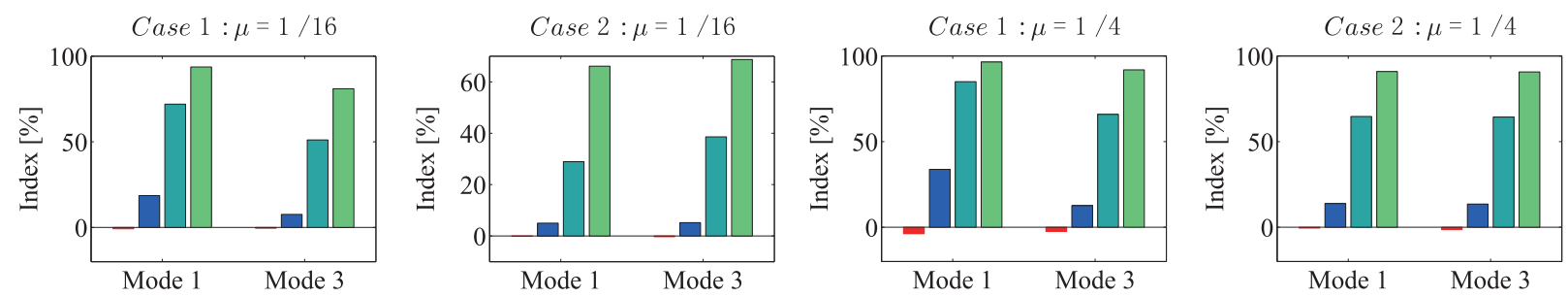

Fig. 9. Index variation in the first and second case for the first and third mode, calculated using frequency ( $\square$ ) or active damping for the three ponderations ( $\left.\square: Q_{1}, \square: Q_{2}, \varpi: Q_{3}\right)$ and two cracks depth $(\mu=1 / 16$ and $\mu=1 / 4)$.

frequency shift included in the matrix $\mathbf{A}_{s}$ leads to disturb the modal control in the mode targeting. And as the control gains are not updated, the controller dynamics is changed. The proposed method is well adapted to detect small cracks increasing the weighting matrices and consequently the control voltage.

\section{Conclusion}

In this paper a new method to detect small cracks based on piezoelectric components and active modal damping is proposed. The originality of the proposed strategy is to exploit the fact that modal control is little robust. The main purpose of the control system is here to detect cracks. Consequently, the control algorithm is designed in order to detect cracks and the weighting matrix is chosen only to this task. Some weighting matrices can be tested by switching the controller between several values up to the crack detection. As the robustness of the controller is inversely proportional to the controller performances, the weighting coefficients must be increase in the case of small cracks. This method does not affect here the structure stability because the system is more and more damped. In other applications, the crack detection could induce some vibration amplification in state of damping but if the 
weighting matrices are tested increasingly, cracks must be detected before a too large amplification. Therefore, it can be applied to monitor the structure dynamics and to detect cracks instead of reduce vibration. The model of the uncracked structure, contained in the Luenberger observer permits to monitor the cracked structure dynamics. The main advantage of the use of a LQG controller to detect cracks is the possibility to use high control voltage in the case of small crack depth when the frequency shift cannot be easily measured. The proposed method is validated with numerical simulation on a clamped-clamped cracked beam including four piezoelectric transducers linked by the LQG control loop. This approach could be extended to plates and more complex industrial systems. Weighting matrix coefficients could be optimize to detect small cracks using a minimum of energy. Moreover, the proposed method can be easily implemented with actuators and sensors traditionally used to detect cracks with frequency shift by adding a controller and an identification algorithm adapted to highly damped controlled systems.

\section{References}

[1] U. Andreaus and P. Baragatti, Experimental damage detection of cracked beams by using nonlinear characteristics of forced response, Mechanical Systems and Signal Processing 31 (2012), 382-404.

[2] M.A. Al-Shudeifat and E.A. Butcher, New breathing functions for the transverse breathing crack of the cracked rotor system: Approach and subcritical harmonic analysis, Journal of Sound and Vibration 31 (2011), 526-544.

[3] M. Athans, The role and use of the stochastic linear-quadratic-gaussian problem in control system design, Automatic Control IEEE Transaction on 16 (1971), 529-552.

[4] A. Badel, D. Guyomar, E. Lefeuvre and C. Richard. Piezoelectric energy harvesting using a synchronized switch technique, Journal of Intelligent Material and Structures 17 (2006), 831-839.

[5] A. Benjeddou, Advances in piezoelectric finite element modeling of adaptive structural elements: A survey, Computers and Structures $\mathbf{7 6}$ (2000), 347-363.

[6] B. Chomette, D. Remond, S. Chesne and L. Gaudiller, Semi-adaptive modal control of on-board electronic boards using identification method, Smart Material and Structures 17 (2008), 1-8.

[7] B. Chomette, S. Chesne, D. Remond and L. Gaudiller, Damage reduction of on-board structures using piezoelectric components and active modal control - application to a printed circuit board, Mechanical System and Signal Processing 24 (2010), 390-397.

[8] B. Chomette and J.-J. Sinou, Crack detection based on optimal control, Journal of Vibration and Control 18 (2012), 1737-1749.

[9] T.G. Chondros, Vibration of a beam with a breathing crack, Journal of Sound and Vibration 239 (2001), 57-67.

[10] S. Christides and A.D.S. Barr, One-dimensional theory of cracked Bernoulli-Euler beams, International Journal of Mechanical Sciences 26 (1984), 639-648.

[11] M. Dilena and A. Morassi, Identification of crack location in vibrating beams from changes in node positions, Journal of Sound and Vibration 255 (2002), 915-930.

[12] B. Faverjon and J.-J. Sinou, Identification of an open crack in a beam by using a posteriori error estimator on the frequency response functions with noisy measurements, European Journal of Mechanics A-Solid 28 (2009), 75-85.

[13] A. Fernandes and J. Pouget, Analytical and numerical modelling of laminated composites with piezoelectric elements, Journal of Intelligent Material Systems and Structures 15 (2004), 753-761.

[14] M.I. Friswell and J.E.T. Penny, Crack modeling for structural health monitoring, Structural Health Monitoring 1 (2002), 139-148.

[15] L. Gaudiller and J.D. Hagopian, Active control of flexible structures using a minimum number of components, Journal of Sound and Vibration 193 (1996), 713-741.

[16] Z. Gosiewski and A.P. Koszewnik, Fast prototyping for the active vibration damping system of mechanical structures, Mechanical Systems and Signal Processing (2012), in press.

[17] N.W. Hagood and A. von Flotow, Damping of structural vibrations with piezoelectric materials and passive electrical networks, Journal of Sound and Vibration 146 (1991), 243-268.

[18] S. Hurlebaus, U. Stöbener and L. Gaul, Vibration reduction of curved panels by active modal control, Computer and Structures 86 (2008), 251-257.

[19] F. Ismail, A. Ibrahim and H.R. Martin, Identification of fatigue cracks from vibration testing, Journal of Sound and Vibration 140 (1990), 305-317.

[20] Z. Kulesza and J. Sawicki, Rigid finite element model of a cracked rotor, Journal of Sound and Vibration 331 (2012), 4415-4169.

[21] D. Luenberger, An introduction to observers, Automatic Control, IEEE Transactions on 16 (1971), 596-602.

[22] V. Piefort, Finite Element Modelling of Piezoelectric Active Structures, Ph.D. Dissertation, Université libre de Bruxelles, 2001.

[23] A. Preumont, Vibration Control of Active Structures: An introduction, second ed., Springer, 2002.

[24] N. Pugno and C. Surace, Evaluation of the non-linear dynamic response to harmonic excitation of a beam with several breathing cracks, Journal of Sound and Vibration 235 (2000), 749-762.

[25] H. Richardson, Mark and D.L. Formenti, Global curve fitting frequency response measurements using the rational fraction polynomial method, in: Proceeding of the International Modal Analysis Conference and Exhibit (1985), 390-397.

[26] P.F. Rizos and N. Aspragathos, Identification of crack location and magnitude in a cantilever beam from the vibration modes, Journal of Sound and Vibration 183 (1990), 381-388.

[27] P. Sadilek and R. Zemcik, Frequency response analysis of hybrid piezoelectric cantilever beam, Engineering Mechanics 17 (2010), $73-82$.

[28] O.S. Salawu, Detection of structural damage through changes in frequency: A review, Engineering Structures 19 (1997), 718-723. 
[29] J.T. Sawicki, M.I. Friswell, Z. Kulesza, A. Wroblewski and J.D. Lekki, Detecting cracked rotors using auxiliary harmonic excitation, Journal of Sound and Vibration 330 (2011), 1365-1381.

[30] J.T. Sawicki and Z. Kulesza, A new observer based method for rotor crack detection, Advances in Vibration Engineering 11 (2012), 131-141.

[31] J.K. Sinha, M.I. Friswell and S. Edwards, Simplified models for the location of cracks in beam structures using measured vibration data, Journal of Sound and Vibration 251 (2002), 13-38.

[32] U. Stöbener and L. Gaul, Active vibration control of a car body based on experimentally evaluated modal parameters, Mechanical Systems and Signal Processing 15 (2001), 173-188. 

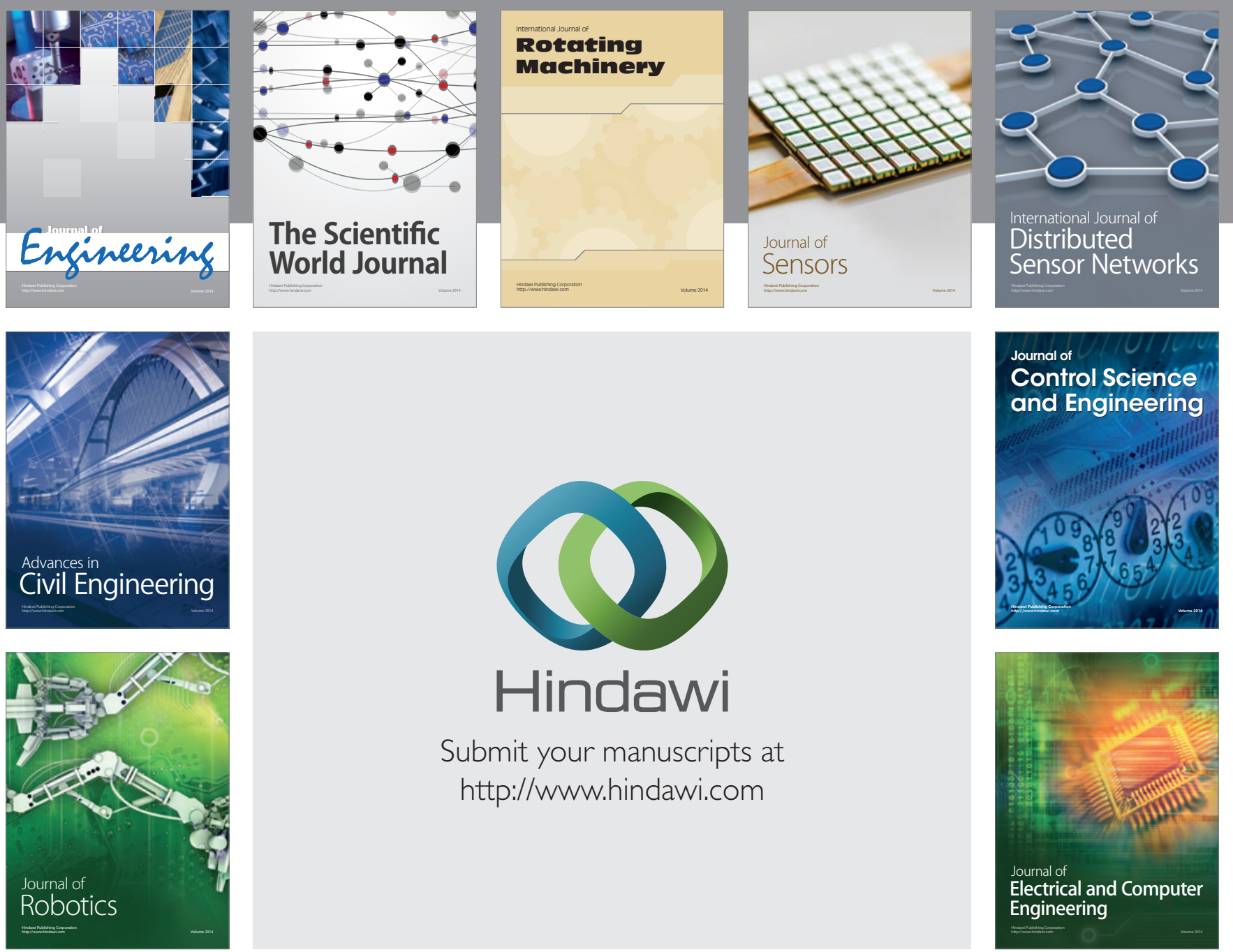

Submit your manuscripts at

http://www.hindawi.com
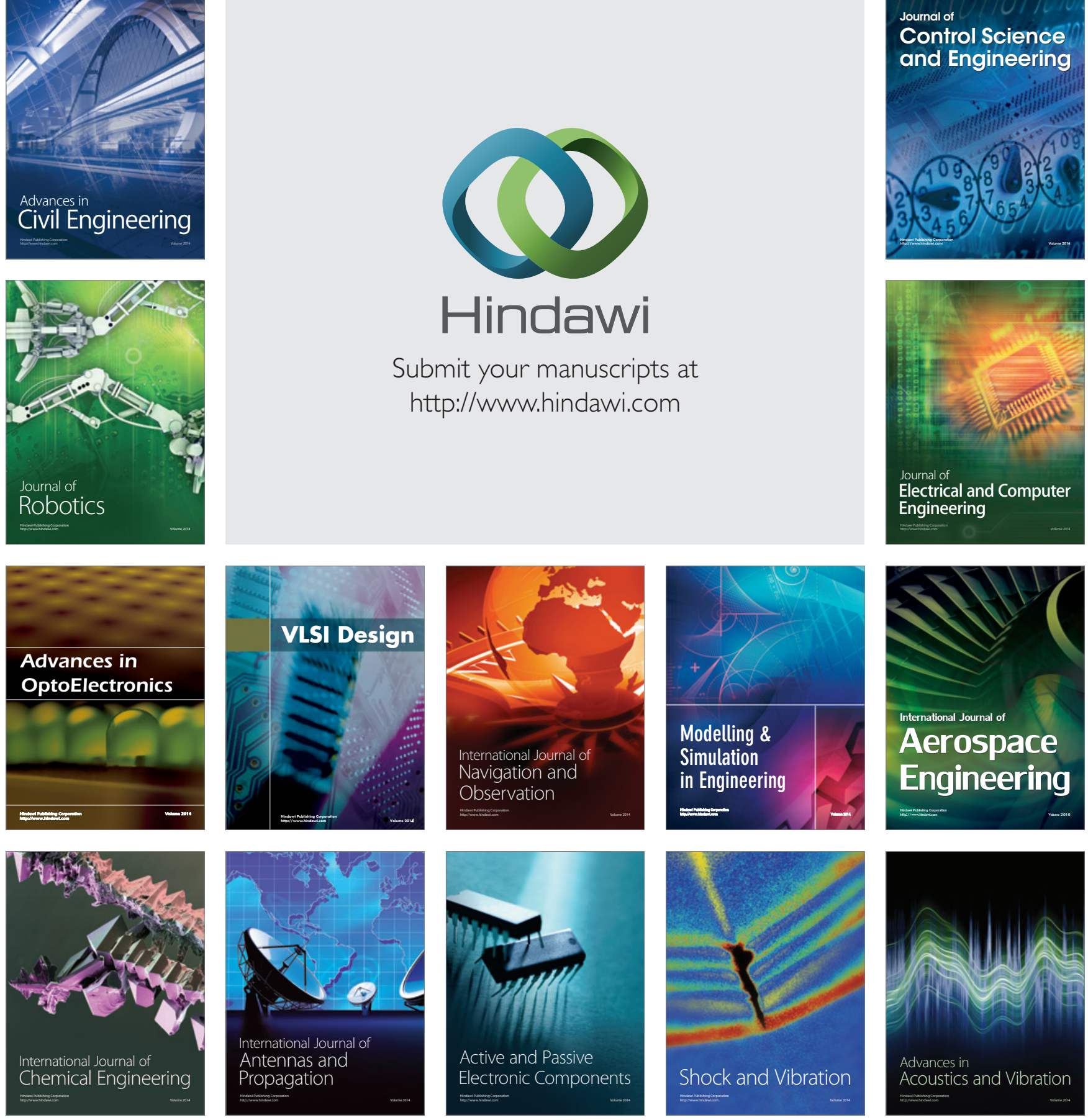